\title{
Reorientation transition of the magnetic proximity polarization in $\mathrm{Fe} /(\mathrm{Ga}, \mathrm{Mn}) \mathrm{As}$ bilayers
}

\author{
M. Sperl, ${ }^{1}$ P. Torelli, ${ }^{2}$ F. Eigenmann, ${ }^{1}$ M. Soda,${ }^{1}$ S. Polesya,${ }^{3}$ M. Utz, ${ }^{1}$ D. Bougeard,${ }^{1}$ H. Ebert, ${ }^{3}$ \\ G. Panaccione, ${ }^{2}$ and C. H. Back ${ }^{1}$ \\ ${ }^{1}$ Institut für Experimentelle und Angewandte Physik, University of Regensburg, 93040 Regensburg, Germany \\ ${ }^{2}$ Consiglio Nazionale delle Ricerche-Istituto Officina dei Materiali (CNR-IOM), Laboratorio TASC, in Area Science Park, \\ I-34149 Trieste, Italy \\ ${ }^{3}$ Department of Chemistry, Ludwig-Maximilians University Munich, 81377 München, Germany \\ (Received 29 August 2011; revised manuscript received 31 January 2012; published 25 May 2012)
}

\begin{abstract}
Recently, it has been observed that thin ferromagnetic Fe films deposited on top of ( $\mathrm{Ga}, \mathrm{Mn})$ As layers induce a significant proximity polarization in the $(\mathrm{Ga}, \mathrm{Mn}) \mathrm{As}$ film even at room temperature. Furthermore, it was found that a thin interfacial region of the $(\mathrm{Ga}, \mathrm{Mn}) \mathrm{As}$ film is coupled antiferromagnetically to the Fe layer. Here we report a series of combined x-ray magnetic dichroism and superconducting quantum interference device magnetometer measurements for $\mathrm{Fe} /(\mathrm{Ga}, \mathrm{Mn})$ As bilayers where the $(\mathrm{Ga}, \mathrm{Mn})$ As layer thickness is varied between 5 and $50 \mathrm{~nm}$. We find a reorientation transition of the magnetic proximity polarization as a function of the (Ga,Mn)As thickness. The data are compared to results obtained performing $a b$ initio calculations. A varying concentration of $\mathrm{Mn}$ interstitials as a function of $(\mathrm{Ga}, \mathrm{Mn})$ As layer thickness is responsible for this reorientation. Furthermore, exchange bias is studied in the fully epitaxial bilayer system. We find a rather strong ferromagnetic exchange bias. The strength of the exchange bias can be estimated by using a simple partial domain wall model.
\end{abstract}

DOI: 10.1103/PhysRevB.85.184428

PACS number(s): 75.50.Pp, 75.30.Et, 78.20.Ls

The diluted magnetic semiconductor (DMS) GaMnAs is a promising material for semiconducting spintronic devices. ${ }^{1}$ Since its first synthesis by Ohno et al., progress has been made both in the understanding of the carrier-mediated mechanisms of the ferromagnetic (FM) state ${ }^{2-4}$ and in the ability to raise the Curie temperature $T_{C}$, mainly by postgrowth annealing. ${ }^{5}$ However, the record values of the Curie temperature seem to stagnate below $200 \mathrm{~K}^{6}$ with no clear strategy to further increase it above room temperature.

A novel route toward increasing the ordering temperature is to engineer the material using ferromagnetic metal overlayers. Tuning the magnetic properties at FM metal/DMS-based interfaces based on the most representative DMS system $(\mathrm{Ga}, \mathrm{Mn}) \mathrm{As}^{7}$ has recently been explored in a variety of experiments. For example, exchange bias is observed in MnAs/(Ga,Mn)As bilayers. ${ }^{8-10}$ In contrast, NiFe/(Ga,Mn)As bilayers show an independent magnetization behavior ${ }^{11}$ with no exchange bias. In the $\mathrm{Fe} /(\mathrm{Ga}, \mathrm{Mn}) \mathrm{As}$ system, the $\mathrm{Fe}$ overlayer induces a proximity polarization antiparallel to the Fe moment within a $1-2 \mathrm{~nm}(\mathrm{Ga}, \mathrm{Mn})$ As region, which is promising for improving the ferromagnetic properties of very thin (Ga,Mn)As films. ${ }^{12-14}$ In fact, we have recently demonstrated that the proximity effect effectively enhances the operation temperature of $\mathrm{Fe} /(\mathrm{Ga}, \mathrm{Mn}) \mathrm{As}$ hybrid spin injection devices when very thin $(\mathrm{Ga}, \mathrm{Mn})$ As injector and detector contacts are capped by an iron layer. ${ }^{15}$

However, to take full advantage of the control of the magnetization of the (Ga,Mn)As injector/detector contacts, it is of importance to understand the coupling mechanism in this epitaxial FM metal/DMS bilayer system. Here we map out the influence of the (Ga,Mn)As thickness in fully epitaxial $\mathrm{Fe} /(\mathrm{Ga}, \mathrm{Mn}) \mathrm{As}$ heterostructures on the interfacial coupling as well as on the magnetization properties of the bulk layer by a combined study using element-specific x-ray magnetic circular dichroism (XMCD) measurements and bulk-sensitive superconducting quantum interference device (SQUID) magnetometry. By means of XMCD we are able to identify a thickness dependent reorientation transition of the Mn magnetization from antiparallel to parallel alignment. Moreover, temperature-dependent XMCD and SQUID measurements show that for $(\mathrm{Ga}, \mathrm{Mn})$ As layer thicknesses below $15 \mathrm{~nm}$ the DMS system is fully ferromagnetically coupled to the Fe. For thicker (Ga,Mn)As layers we are able to identify a ferromagnetic exchange bias effect due to the presence of $\mathrm{Fe}$ on (Ga,Mn)As.

\section{EXPERIMENTAL DETAILS}

For the experiments (Ga,Mn)As films were grown on semi-insulating (001) GaAs substrates by LT MBE using a modified Veeco Gen II system. After heating of the epiready substrates to $600{ }^{\circ} \mathrm{C}$ in UHV to remove water and oxide from the surface, a 10-nm-thick LT-GaAs buffer layer was grown $\left(T \approx 200^{\circ} \mathrm{C}\right)$. Subsequently, the $(\mathrm{Ga}, \mathrm{Mn})$ As layers were deposited with Mn concentrations of $x=0.06$ or $x=$ 0.12 and different thicknesses from 3 to $35 \mathrm{~nm}$. The growth rate for the low-temperature growth was below $0.3 \AA / \mathrm{s}$. During deposition the growth temperature as well as the $\mathrm{As}(\mathrm{Ga}+\mathrm{Mn})$ ratio were kept constant at $T_{\text {growth }}=234{ }^{\circ} \mathrm{C}, \mathrm{As} /(\mathrm{Ga}+\mathrm{Mn})=$ $3.1: 1$ for $6 \% \mathrm{Mn}$ and $T_{\text {growth }}=181^{\circ} \mathrm{C}, \mathrm{As} /(\mathrm{Ga}+\mathrm{Mn})=1.0: 1$ for $12 \% \mathrm{Mn}$. The nominal Mn concentration was determined by secondary ion mass spectroscopy (SIMS) measurements. The absolute $\mathrm{Ga}$ deposition rate was determined by reflection high-energy diffraction (RHEED) oscillation on (001) GaAs. Details on the (Ga,Mn)As growth and on the MBE system are given in Refs. 16-18.

Subsequently the samples were transferred to an attached metal MBE system without breaking the vaccum and $1.5 \mathrm{~nm}$ of Fe was epitaxially grown on all samples at room temperature to avoid interdiffusion. The Fe growth rate was controlled in situ by quartz monitors. Finally, the sample was covered with $4 \mathrm{~nm}$ of $\mathrm{Au}$ to prevent oxidation. By use of a mechanical shutter a part of the 2-inch wafer is covered during deposition of both metals to define an uncovered $(\mathrm{Ga}, \mathrm{Mn})$ As reference 
area. The epitaxial quality was confirmed by cross-sectional transmission electron microscopy (TEM) studies revealing a smooth interface between Fe and (Ga,Mn)As.

The static magnetic properties of both the Fe/(Ga,Mn)As heterostructure and the $(\mathrm{Ga}, \mathrm{Mn}) \mathrm{As}$ reference layer were determined using a SQUID magnetometer. Moreover, XMCD experiments at the $L_{2,3}$ edges of $\mathrm{Fe}$ and $\mathrm{Mn}$, on $\mathrm{Fe} /(\mathrm{Ga}, \mathrm{Mn}) \mathrm{As}$ samples of different thicknesses, were carried out at the APE beamline of the Elettra Synchrotron (Trieste, Italy). The XMCD spectra as a function of the magnetization direction were acquired in total electron yield mode (TEY) by recording the drain current at remanence. The magnetization direction was reversed at each point of the XMCD spectra. During the experiment the incident light formed an angle of $45^{\circ}$ with respect to the surface normal. Chemically resolved magnetic hysteresis loops of Fe and $\mathrm{Mn}$ were obtained by recording the value of the $L_{3}$ edge as a function of the applied magnetic field. This signal was subsequently divided by the same measurement obtained by fixing the photon energy $10 \mathrm{eV}$ lower than the $L_{3}$ edge in order to remove artifacts due to influence of the stray fields on the secondary electrons. Both XMCD spectra and magnetic hysteresis loops were measured with opposite photon helicities, resulting in an inversion of the measured signals. In order to obtain a detectable signal of $\mathrm{Fe}$ and $\mathrm{Mn}$ in TEY, the sample was partially decapped from Au by controlled Ar-Ion sputtering in situ prior to the experiments. All samples were measured at room temperature, and the $\mathrm{Fe} / 5 \mathrm{~nm}(\mathrm{Ga}, \mathrm{Mn})$ As sample was also measured at $40 \mathrm{~K}$.

\section{RESULTS AND DISCUSSION}

\section{A. X-ray magnetic circular dichroism}

In this experiment we measured XMCD spectra of $\mathrm{Fe} /(\mathrm{Ga}, \mathrm{Mn})$ As samples with different thicknesses of the DMS film (ranging from 3 to $35 \mathrm{~nm}$ ) and with different $\mathrm{Mn}$ concentrations, namely $6 \%$ and $12 \%$. Measurements were performed at room temperature and at $40 \mathrm{~K}$. In all samples the Fe film displays a large normalized XMCD signal around $30 \%$ at the $L_{3}$ edge after correction for the incident angle and degree of light polarization, indicating that the Fe layer is ferromagnetic and fully magnetized at remanence. The Mn $L_{2,3}$ edges display a ferromagnetic XMCD signal at remanence in all measured samples as well, with a smaller XMCD signal with respect to Fe. The presence of a clear Mn magnetic signal at room temperature indicates that a small part of the (Ga,Mn)As layer, located at the interface, exhibits ferromagnetic order due to the proximity effect of the Fe layer, as already observed in previous experiments. ${ }^{12-14}$ In Fig. 1 XMCD spectra are displayed, which were recorded at room temperature at the Mn [Fig. 1(a)] and Fe [Fig. 1(b)] $L_{2,3}$ edges of a sample of $5 \mathrm{~nm}$ of (Ga,Mn)As thickness and $12 \%$ of $\mathrm{Mn}$ concentration. It is clearly visible that $\mathrm{Fe}$ displays the typical dichroism of bulk Fe with an normalized asymmetry value [defined as $\left(I^{+}-I^{-}\right) /\left(I^{+}+I^{-}\right)$at the $L_{3}$ edge] close to $30 \%$ while Mn shows only a small dichroism of about $2 \%$. Even though the magnitude and the shape of the XMCD of $\mathrm{Fe}$ and $\mathrm{Mn}$ is in perfect agreement with previous studies the sign of the coupling between the $\mathrm{Fe}$ and $\mathrm{Mn}$ is opposite to the one observed so far. In fact, an antiparallel alignment has always been observed at the $\mathrm{Fe} /(\mathrm{Ga}, \mathrm{Mn}) \mathrm{As}$ interface, while the up-down feature of the XMCD of Fe and Mn in Figs. 1(a) and 1(b) clearly demonstrates parallel alignment of the $\mathrm{Fe}$ and $\mathrm{Mn}$ moments. This peculiar finding calls for further investigation of this effect. Indeed, the main difference between the sample studied here and previously studied samples is the thickness of the $(\mathrm{Ga}, \mathrm{Mn})$ As layer. Consequently, we performed a $(\mathrm{Ga}, \mathrm{Mn}) \mathrm{As}$ thickness-dependent XMCD study. To this end we measure a set of fully epitaxial $\mathrm{Fe} /(\mathrm{Ga}, \mathrm{Mn}) \mathrm{As}$ samples with variable (Ga,Mn)As thickness (3, 5, 8, 10, 15, and $35 \mathrm{~nm}$ ) and with a Mn concentration of $6 \%$ as well as $5 \mathrm{~nm}$ (Ga,Mn)As with films $12 \% \mathrm{Mn}$. The results are summarized in Fig. 1(c). Here we report the Mn XMCD asymmetry values where the negative and positive values indicate antiparallel and parallel alignment of the Mn magnetic moments with respect to the Fe moments, respectively. For $(\mathrm{Ga}, \mathrm{Mn}) \mathrm{As}$ films thicker than $15 \mathrm{~nm}$ we observe antiparallel coupling but for films of $10 \mathrm{~nm}$ and thinner the sign of the coupling is reversed and the coupling between the $\mathrm{Fe}$ and $\mathrm{Mn}$ moment is now ferromagnetic. We, further, investigate the properties of the ferromagnetic coupling as a function of an applied magnetic field along [110] direction and as a function of the temperature by measuring XMCD spectra at $40 \mathrm{~K}$ as well as chemically selective hysteresis loops. The XMCD spectra of Fe and Mn of the $12 \% \mathrm{Mn}$ doped sample $5 \mathrm{~nm}$ thick $(\mathrm{Ga}, \mathrm{Mn})$ As recorded
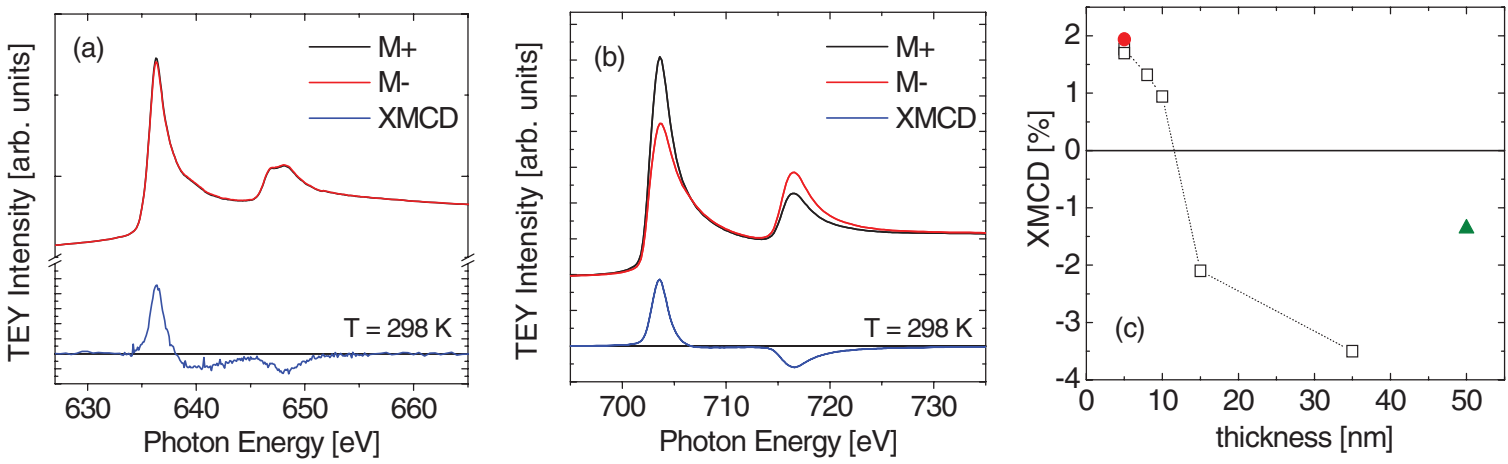

FIG. 1. (Color online) Fe and Mn $L_{2,3}$ XAS/XMCD spectra recorded at room temperature for Fe/(Ga,Mn)As heterostructures with $12 \%$ Mn: (a) Fe/5 nm (Ga,Mn)As measured at the Mn $L_{2,3}$ edge. (b) Fe/5 nm (Ga,Mn)As measured at the Fe $L_{2,3}$ edge. (c) Normalized Mn $L_{3}$ dichroism as a function of (Ga,Mn)As thickness for $6 \% \mathrm{Mn}$ (black square) and for $5 \mathrm{~nm}(\mathrm{Ga}, \mathrm{Mn}) \mathrm{As}, 12 \%$ Mn (red circle). The green triangle denotes the induced dichroism for $50 \mathrm{~nm}(\mathrm{Ga}, \mathrm{Mn}) \mathrm{As}$ with $6 \% \mathrm{Mn}$ (data taken from measurements of Ref. 13). 

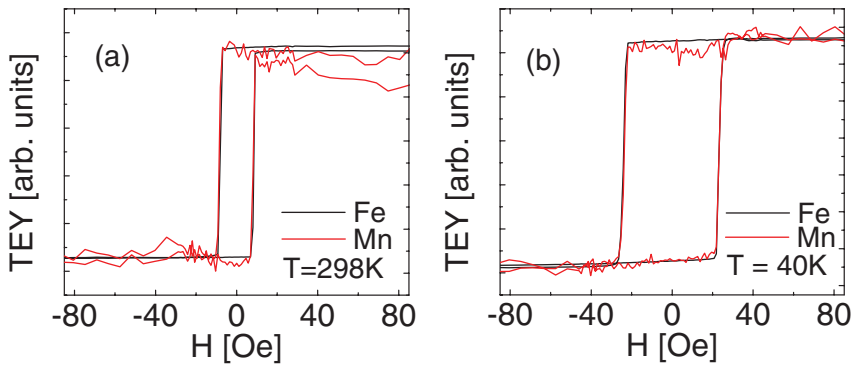

FIG. 2. (Color online) Element specific hysteresis loops with an in-plane field along [110] direction: (a) XMCD hysteresis loop measured at the Mn $L_{3}$ edge (red line) for $\mathrm{Fe} / 5 \mathrm{~nm}(\mathrm{Ga}, \mathrm{Mn}) \mathrm{As}$ with $12 \% \mathrm{Mn}$ at room temperature normalized to the hysteresis loop measured at the Fe $L_{3}$ edge (black line). (b) XMCD hysteresis loop measured at the $\mathrm{Mn} L_{3}$ edge (red line) normalized to the hysteresis loop measured at the $\mathrm{Fe} L_{3}$ edge (black line) for the same sample at $T=40 \mathrm{~K}$.

at $40 \mathrm{~K}$ are identical to the ones measured at room temperature except for the magnitude of the Mn XMCD asymmetry which is about $6 \%$ at low temperature. This is easily explained by the reduction of magnetization due to thermal fluctuations. In Figs. 2(a) and 2(b) the hysteresis loops recorded at the $L_{3}$ edges of $\mathrm{Fe}$ and $\mathrm{Mn}$ at room temperature and $40 \mathrm{~K}$ are reported. Fe displays a square hysteresis with coercive fields of $8 \mathrm{Oe}$ at room temperature that increase up to 24 Oe at $40 \mathrm{~K}$. The Mn signal (which has been normalized to the Fe signal for better comparison) is a perfect copy of the iron signal demonstrating that the Mn polarization is totally dominated by the Fe magnetization.

To substantiate the experimentally observed ferromagnetic alignment of $\mathrm{Fe}$ and $\mathrm{Mn}$ moments we have performed a firstprinciples study of the $\mathrm{GaAs} /\left(\mathrm{Ga}_{1-x} \mathrm{Mn}_{x}\right) \mathrm{As} / \mathrm{Fe}$ system using the spin polarized relativistic tight-binding KKR Green's function method. ${ }^{19}$ Exchange and correlation were treated within the framework of the local spin density approximation (LSDA) to the density-functional theory, using the parametrization of Vosko, Wilk, and Nusair. ${ }^{20}$ The coherent potential approximation (CPA) is used to describe the substitutional disorder in (Ga,Mn)As. ${ }^{21,22}$ This allows us to avoid time-consuming supercell calculations and to use in the present case only two atoms per layer to construct the (Ga,Mn)As (001) surface. The calculations were performed for a semi-infinite geometry: eight monolayers of $\left(\mathrm{Ga}_{1-x} \mathrm{Mn}_{x}\right)$ As deposited on the GaAs (001) surface and covered with a one monolayer thick Fe film. The concentration $x$ of Mn on the Ga sites was taken to be $5 \%$.

Five different conditions on the interface with $\mathrm{Fe}$ were considered as follows: I, Ga terminated; II, Ga terminated with Mn interstitials at the interface; III, As terminated; IV, As terminated with $\mathrm{Mn}$ interstitials at the interface; and $\mathrm{V}$, interface consisting of a single atomic plane of alternating $\mathrm{Fe}$ and As atoms. ${ }^{23}$ On the basis of the obtained electronic structure the exchange interactions $J_{i j}$ between magnetic atoms have been calculated using an approach suggested by Liechtenstein et al.. ${ }^{24}$
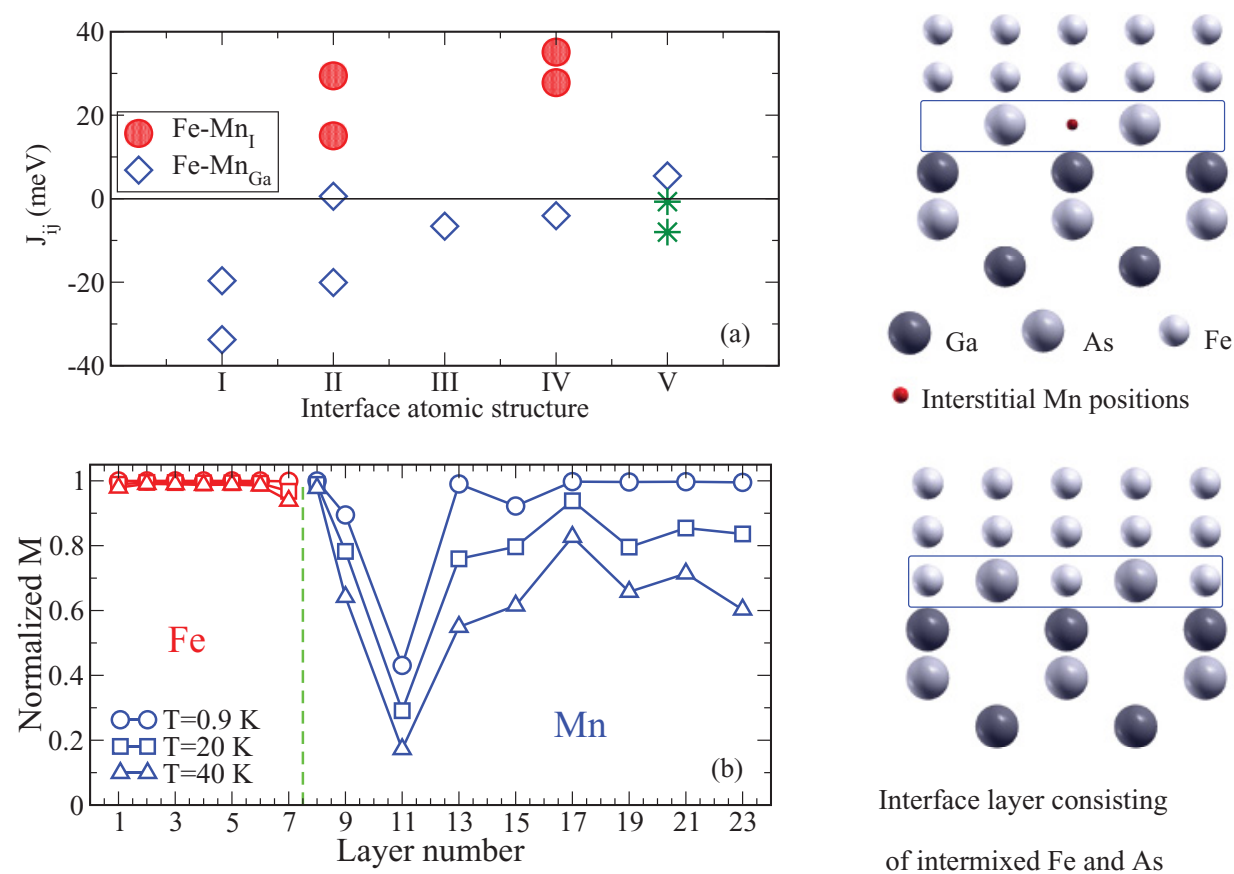

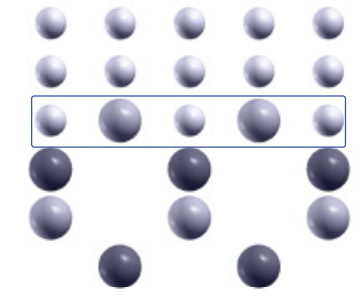

Interface layer consisting

of intermixed $\mathrm{Fe}$ and $\mathrm{As}$ (c)

(d)

FIG. 3. (Color online) (a) Exchange coupling parameters $J_{i j}$ between $\mathrm{Fe}$ and $\mathrm{Mn}$ atoms for five different atomic structures at the (Ga,Mn)As/Fe interface: I, Ga terminated; II, Ga terminated with Mn interstitials at the interface; III, As terminated; IV, As terminated with $\mathrm{Mn}$ interstitials at the interface; and $\mathrm{V}$, interface consisting of a single atomic plane of alternating Fe and As atoms. ${ }^{23}$ The stars $(*)$ for structure $\mathrm{V}$ correspond to the interactions of $\mathrm{Mn}_{\mathrm{Ga}}$ from the interface with $\mathrm{Fe}$ atoms from the first fully occupied $\mathrm{Fe}$ layer. In each case, the parameters are given for the nearest-neighbor $\mathrm{Mn}_{\mathrm{Ga}}-\mathrm{Fe}\left(\mathrm{Mn}_{\mathrm{I}}-\mathrm{Fe}\right)$ distances. (b) Results of Monte Carlo calculations: layer-resolved magnetization for a 7-ML Fe/8-ML $\left(\mathrm{Ga}_{0.95} \mathrm{Mn}_{0.05} \mathrm{As}\right)$ As bilayer with an As-terminated interface and Mn interstitials at the interface layer of (Ga,Mn)As (atomic structure IV). Each atomic layer is counted. In this case layer 8 contains As atoms. (c) Structure IV, the frame is around the interface layer. (d) Structure V, the AsFe layer at the interface is shown with the frame. 
The results for the exchange interactions between $\mathrm{Fe}$ and $\mathrm{Mn}$ atoms at the interface are presented in Fig. 3(a). As one can observe that, for the Ga-terminated interface with only substitutional Mn (structure I), the exchange coupling between $\mathrm{Mn}_{\mathrm{Ga}}$ (Mn on Ga sites) and $\mathrm{Fe}$ atoms at the interface is strongly antiferromagnetic. Moreover, the coupling between $\mathrm{Mn}_{\mathrm{Ga}}$ within the (Ga,Mn)As film is ferromagnetic. As it was shown earlier (Ref. 11), this results in ferromagnetic order within the (Ga,Mn)As layers close to the Fe film up to room temperature. In the case of an As-terminated interface (structure III), the exchange interaction between $\mathrm{Fe}$ and $\mathrm{Mn} \mathrm{Ga}$ atoms at the interface is also antiferromagnetic but weaker than in the case of a Ga termination. The magnetic coupling between $\mathrm{Mn}_{\mathrm{Ga}}$ for the two layers close to the Fe films in this case is antiferromagnetic. The strength of this coupling is $\sim 15.6 \mathrm{meV}$ for $\mathrm{Mn}_{\mathrm{Ga}}$ atoms lying within the same plane and $\sim 11.5 \mathrm{meV}$ for $\mathrm{Mn}$ from the neiboring planes. As a result, a disordered noncollinear magnetic structure is created within the $(\mathrm{Ga}, \mathrm{Mn}) \mathrm{As}$ layers close to the Fe film. Note that experimentally a mix of Asand Ga-terminated surfaces is most likely. In this case one can expect antiferromagnetic alignment of the average $(\mathrm{Ga}, \mathrm{Mn}) \mathrm{As}$ magnetization close to the interface with respect to the Fe film magnetization.

Theoretically, a different idealized interface structure was considered that is not observed experimentally. This surface structure consists of a single atomic plane of alternating Fe and As atoms [Fig. 3(d)], structure V in Fig. 3(a). The exchange coupling between $\mathrm{Fe}$ and $\mathrm{Mn}_{\mathrm{Ga}}$ at the interface have rather small values in this case and the ferromagnetic $\mathrm{Mn}_{\mathrm{Ga}}-\mathrm{Fe}$ interaction with $\mathrm{Fe}$ atoms from the interface AsFe layer is compensated by the antiferromagnetic $\mathrm{Mn}_{\mathrm{Ga}}-\mathrm{Fe}$ coupling with $\mathrm{Fe}$ from the next to the interface fully occupied Fe layer. Thus, we can conclude that these interface atomic structures cannot be responsible for the ferromagnetic alignment of $\mathrm{Fe}$ and (Ga,Mn)As layers.

The exchange interaction changes strongly if Mn interstitials $\left(\mathrm{Mn}_{\mathrm{I}}\right)$ are present at the interface layer of $\mathrm{Fe} /(\mathrm{Ga}, \mathrm{Mn}) \mathrm{As}$. The $\mathrm{Mn}$ atoms in the interstitial positions interact ferromagnetically with $\mathrm{Fe}$ atoms for both cases of $\mathrm{Ga}$ - and Asterminated interfaces [Fig. 3(a), structures II and IV). At the same time, the interaction between substitutional Mn with $\mathrm{Fe}$ is still antiferromagnetic but it is weaker. Despite the strong antiferromagnetic coupling between nearest-neighbor $\mathrm{Mn}_{\mathrm{Ga}}$ and $\mathrm{Mn}_{\mathrm{I}}$ magnetic moments, the orientation of the total Mn magnetic moment of the interface layer depends significantly on $\mathrm{Mn}-\mathrm{Fe}$ interactions. As a result, ferromagnetic alignment of the $\mathrm{Fe}$ and $\mathrm{Mn}$ atoms at the interface will prevail. This is confirmed by Monte Carlo calculations. In Fig. 3(b) the layer-resolved normalized magnetization $M$ for the Asterminated interface with $\mathrm{Mn}_{\mathrm{I}}$ at the interface is shown for three temperatures, $0.9,20$, and $40 \mathrm{~K}$. The concentration of $\mathrm{Mn}_{\mathrm{I}}$ in the interface layer is equal to $10 \%$ and there is no interstitial $\mathrm{Mn}$ present in other layers. The concentration of $\mathrm{Mn}_{\mathrm{Ga}}$ is $5 \%$. All $\mathrm{Mn}$ atoms are randomly distributed on terahedral interstitial (for the interface layer) or Ga substitutional positions. The thermodynamic averaging was performed over 10 different disorder configurations. As one can see, the magnetization of Fe and $(\mathrm{Ga}, \mathrm{Mn}) \mathrm{As}$ films is aligned ferromagnetically. The reduced values of magnetization for layers 9 and 11 (corresponding to the first and second layers containing $\mathrm{Mn}_{\mathrm{Ga}}$ ) is a consequence of the disordered magnetic structure due to the $\mathrm{Mn}_{\mathrm{Ga}}-\mathrm{Mn}_{\mathrm{I}}$ and $\mathrm{Mn}_{\mathrm{Ga}}-\mathrm{Mn}_{\mathrm{Ga}}$ antiferromagnetic interactions in these layers. The transition to a paramagnetic state for the $(\mathrm{Ga}, \mathrm{Mn})$ As film occurs at around $50 \mathrm{~K}$, while the layer nearest to the Fe layer (contaning Mn interstitials) keeps ferromagnetic order well above room temperature due to the strong coupling to the Fe film. For the Ga-terminated interface ferromagnetic alignment of the average magnetization of (Ga,Mn)As and Fe films was also obtained. But, in this case, the disordered magnetic structure that reduces the average magnetization is established in the $(\mathrm{Ga}, \mathrm{Mn})$ As nearest to the Fe film. It should be also mentioned that for the As-terminated interface the concentration of $5 \%$ of $\mathrm{Mn}_{\mathrm{I}}$ could result in ferromagnetic orientation of the average magnetization of a (Ga,Mn)As layers with respect to the Fe film magnetization. The obtained results are fully consistent with the experimental data and ferromagnetic alignment between $\mathrm{Mn}$ and $\mathrm{Fe}$ atoms is explained by the presence of interstitial $\mathrm{Mn}$ in the vicinity of the Fe film.

We attribute the observed change in relative orientation of magnetization at the interface between $\mathrm{Fe}$ and $\mathrm{Mn}$ to a change in $\mathrm{Mn}$ interstitial concentration in dependence of the (Ga,Mn)As thickness. For thick (250-300 nm) layers of $(\mathrm{Ga}, \mathrm{Mn})$ As Koeder et al. have found a pronounced decrease of the carrier concentration from the surface toward the $(\mathrm{Ga}, \mathrm{Mn}) \mathrm{As} / \mathrm{GaAs}$ interface due to a nonhomogeneous distribution of point defects like Mn interstitials or As antisites in the layer. ${ }^{25}$ Moreover, it is found that for film thicknesses less than $10 \mathrm{~nm}$, the diffusion length of $\mathrm{Mn}_{\mathrm{I}}$ in the film is comparable to the film thickness. 5,26

For thin (Ga,Mn)As layers this is manifested by a high amount of randomly distributed $\mathrm{Mn}$ at the surface represented by a surface oxide layer. For thicker $(\mathrm{Ga}, \mathrm{Mn})$ As layers, on the other hand, an increasing Mn interstitial concentration away from the surface is found. ${ }^{26}$ In our case, a chemical passivation of $\mathrm{Mn}$ interstitials in a surface oxide layer is not likely since the (Ga,Mn)As film was directly overgrown with $\mathrm{Fe}$ after preparation. This may lead to a significant increased number of $\mathrm{Mn}$ interstitials at the interface between $\mathrm{Fe}$ and $(\mathrm{Ga}, \mathrm{Mn}) \mathrm{As}$ and, consequently, to a reorientation of the induced magnetization at the interface with reduced (Ga,Mn)As thickness.

\section{B. Magnetometry}

For magnetic characterization SQUID magnetometry is performed on $\mathrm{Au} / \mathrm{Fe} /(\mathrm{Ga}, \mathrm{Mn}) \mathrm{As} / \mathrm{GaAs}(001)$ as well as on reference (Ga,Mn)As/GaAs(001) samples for all (Ga,Mn)As thicknesses and Mn concentrations (6\% and 12\%). Figure 4(a) shows a major hysteresis loop at $20 \mathrm{~K}$ for a $\mathrm{Fe} /(\mathrm{Ga}, \mathrm{Mn}) \mathrm{As}$ heterostructures with a (Ga,Mn)As thickness of $30 \mathrm{~nm}$ and Mn concentration of $12 \%$ and field applied along the [110] crystal axis. This corresponds to an easy axis of the $\mathrm{Fe}$ layer for the choosen Fe thickness. The magnetic moment is not normalized to the sample volume since the bilayer stack consists of two different ferromagnetic layers. A distinct two-step switching process is observed for Fe on 20 and $30 \mathrm{~nm}$ (Ga,Mn)As with 6\% $\mathrm{Mn}$ and $\mathrm{Fe} / 30 \mathrm{~nm}(\mathrm{Ga}, \mathrm{Mn})$ As with $12 \%$ $\mathrm{Mn}$ for all temperatures below $T_{C}$ of $(\mathrm{Ga}, \mathrm{Mn}) \mathrm{As}$. Interestingly, the two-step switching is absent in the case of Fe/30 nm 

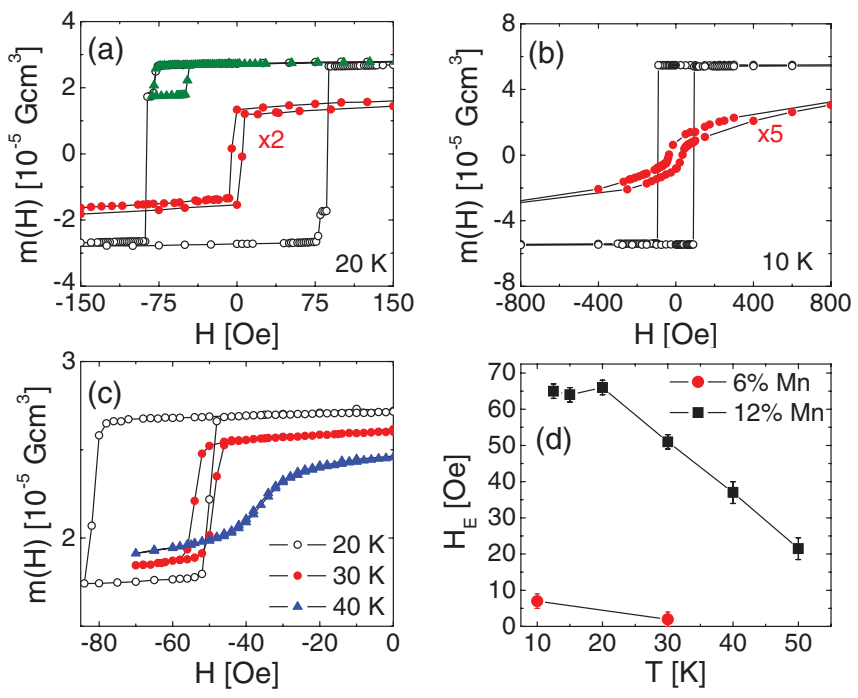

FIG. 4. (Color online) (a) Major magnetization loop $m(H)$ along (Ga,Mn)As [110] measured at $T=20 \mathrm{~K}$ for a bilayer $\mathrm{Fe} / 30 \mathrm{~nm}$ (Ga,Mn)As sample with $12 \% \mathrm{Mn}$ showing a distinct two-step switching process (open circles); minor magnetization loop after saturating the Fe layer (red solid circles). (b) SQUID measurement of the field dependence of the magnetic moment $m(H)$ for $\mathrm{Fe} / 5 \mathrm{~nm}(\mathrm{Ga}, \mathrm{Mn}) \mathrm{As}, 12 \% \mathrm{Mn}$ (open circles) and the corresponding (Ga,Mn)As reference (red solid circles) along [110] measured at $10 \mathrm{~K}$. (c) Minor magnetization loops for Fe/30 nm (Ga,Mn)As with 12\% Mn measured along [110] for different temperatures. (d) Dependence of the exchange field $H_{E}$ on temperature for $\mathrm{Fe} / 30 \mathrm{~nm}(\mathrm{Ga}, \mathrm{Mn}) \mathrm{As}$ with $12 \% \mathrm{Mn}$ (black squares) and $6 \% \mathrm{Mn}$ (red circle).

(Ga,Mn)As with $12 \% \mathrm{Mn}$ at the lowest measuring temperature $(10 \mathrm{~K})$. By comparison to the $(\mathrm{Ga}, \mathrm{Mn})$ As reference sample and hysteresis loops above $T_{C}$ of $(\mathrm{Ga}, \mathrm{Mn}) \mathrm{As}$, we can assign the smaller coercive field to (Ga,Mn)As and the second switching process to $\mathrm{Fe}$. The situation changes considerably if the (Ga,Mn)As thickness is reduced. Remarkably, for Fe/ $x \mathrm{~nm}$ (Ga,Mn)As ( $x=5,8,10,12,15 \mathrm{~nm})$, no separate switching field for $\mathrm{Fe}$ and $(\mathrm{Ga}, \mathrm{Mn}) \mathrm{As}$ can be observed in the whole temperature range below the $T_{C}$ of $(\mathrm{Ga}, \mathrm{Mn}) \mathrm{As}$. Corresponding data are shown in Fig. 4(b) for Fe/5 nm (Ga,Mn)As with 12\% $\mathrm{Mn}$. The single switching event is determined by the coercive field of the Fe film, which means there is a mutual switching of $\mathrm{Fe}$ and $(\mathrm{Ga}, \mathrm{Mn})$ As defined by the magnetization reversal of Fe. This ferromagnetic coupling between both magnetic layers in the integral SQUID measurements is in agreement with the element-specific XMCD hysteresis measurement (Fig. 2). For the $\mathrm{Fe} / 30 \mathrm{~nm}$ (Ga,Mn)As samples (6\% and 12\% Mn) we measured minor loops as a function of temperature to determine the exchange field. The minor loops were measured by saturating the sample in a positive magnetic field and subsequently demagnetizing the sample in a negative field large enough to switch the (Ga,Mn)As layer but smaller then the coercive field of Fe. Figures 4(a) and 4(c) shows the minor loops for three different temperatures for $\mathrm{Fe} / 30 \mathrm{~nm}$ (Ga,Mn)As with $12 \% \mathrm{Mn}$; clearly a displacement of the center of the minor loop can be observed. The shift is opposite to the magnetization of the Fe layer for all measured temperatures indicating an exchange bias that is ferromagnetic. The observed exchange field is roughly 65 Oe at the lowest measurement temperature of $12.5 \mathrm{~K}$ [Fig. 4(d)] and one order of magnitude smaller for $\mathrm{Fe} / 30 \mathrm{~nm}(\mathrm{Ga}, \mathrm{Mn})$ As with $6 \% \mathrm{Mn}$. The evidence of ferromagnetic exchange bias is seemingly in contrast to our observation of an antiparallel alignment (thickness $(\mathrm{Ga}, \mathrm{Mn}) \mathrm{As} \geqslant 15 \mathrm{~nm}$ ) of the two layers at the interface ${ }^{12,13}$ and to the observation of an antiferromagnetic exchange bias effect in recent SQUID measurements of $\mathrm{Fe} /(\mathrm{Ga}, \mathrm{Mn}) \mathrm{As}$ bilayer samples. ${ }^{14}$ Interestingly, a ferromagnetic coupling was also reported by Wilson et al. for $\mathrm{MnAs} /(\mathrm{Ga}, \mathrm{Mn}) \mathrm{As}^{10}$ but without the possibility to study the relative orientation of magnetization at the interface.

To calculate the exchange field experienced by $(\mathrm{Ga}, \mathrm{Mn}) \mathrm{As}$ in the evaluated bilayer system we use a simple model proposed by Mauri et al. to describe exchange bias systems ${ }^{27}$ and successfully adopted to calculate the exchange field of hard/soft ferromagnetic bilayers. ${ }^{10,28}$ Here it is considered that the magnetization of the Fe layer is fixed and aligned along its easy axis in the positive [110] direction of (Ga,Mn)As. At the interface within a thin layer $(d \approx 1-2 \mathrm{~nm})$ the magnetization of GaMnAs is aligned antiparallel to the Fe magnetization up to a field of $50 \mathrm{kOe}$, as it was deduced from recent measurements. ${ }^{12,13}$ Subsequently, the magnetization of the ( $\mathrm{Ga}, \mathrm{Mn}) \mathrm{As}$ is free to rotate toward the global easy axis of (Ga,Mn)As. In the presence of a cubic and uniaxial anisotropy the direction of the bulk $(\mathrm{Ga}, \mathrm{Mn})$ As easy axis with respect to the [110] direction is given by (the other easy axis is located symmetrically with respect to the [110] direction):

$$
\sin \varphi=\sqrt{\left(K_{C}-K_{U}\right) / 2 K_{C}} .
$$

Here $K_{C}$ and $K_{U}$ denote the cubic and the uniaxial anisotropy constant. We assume that a partial domain wall (PDW) of thickness $t_{1}$ will nucleate in the (Ga,Mn)As film away from the interfacial coupled layer. A sketch for the exchange coupled $\mathrm{Fe} /(\mathrm{Ga}, \mathrm{Mn}) \mathrm{As}$ bilayer is given in Fig. 5.

Considering a thin interfacial layer antiparallel to $\mathrm{Fe}$ and a typical domain wall width of $\sim 40 \mathrm{~nm}^{29}$ we assume $t_{2}, d \ll$ $t_{1} \approx t_{\mathrm{GMA}}\left[t_{\mathrm{GMA}}\right.$ : thickness of the $(\mathrm{Ga}, \mathrm{Mn}) \mathrm{As}$ film]. Using

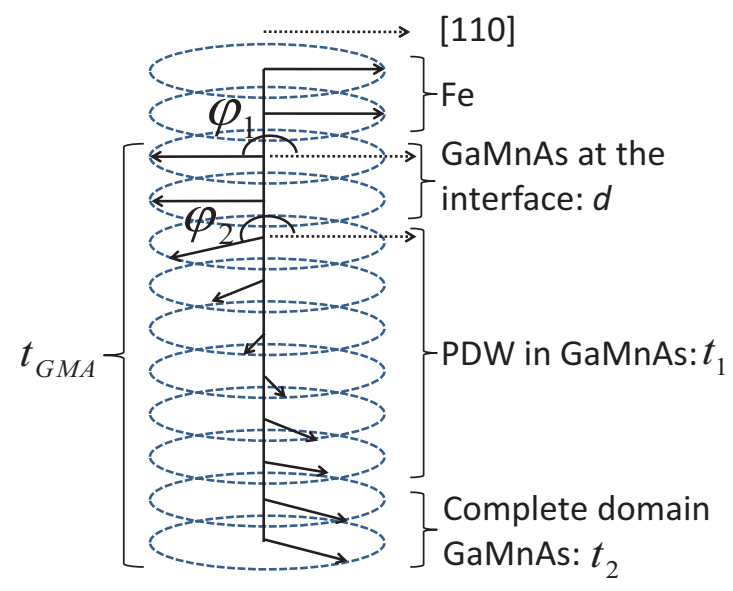

FIG. 5. (Color online) Schematic representation of a partial domain-wall configuration in (Ga,Mn)As. Directly at the interface an interfacial layer with thickness $d$ of $(\mathrm{Ga}, \mathrm{Mn})$ As is aligned antiparallel with respect to $\mathrm{Fe}$. The arrows represent the spin direction, which continuously rotates with increasing distance from the interface. At a certain distance $t_{1}$, a complete domain with thickness $t_{2}$ is formed. 
this approximation the energy density per unit area can be expressed by ${ }^{10}$

$$
\begin{aligned}
E= & 2 \sqrt{A K_{\mathrm{eff}}}\left(\cos \varphi_{1}-\cos \varphi_{2}\right) \\
& -\frac{A_{\mathrm{ex}}}{d} \cos \varphi_{1}+K_{U} t_{\mathrm{GMA}} \sin ^{2} \varphi_{2} \\
& +\frac{1}{4} K_{C} t_{\mathrm{GMA}} \cos ^{2} 2 \varphi_{2}-H M t_{\mathrm{GMA}} \cos \varphi_{2} .
\end{aligned}
$$

The first term represents the energy of the PDW determined by the spin stiffness constant $A$ and the effective anisotropy $K_{\text {eff }}$, and $\varphi_{1}$ is the angle between the magnetization of Fe and the induced magnetization in (Ga,Mn)As at the interface and $\varphi_{2}$ is the angle between the Fe layer and the easy axis of the bulk GaMnAs. The second term $\left(A_{\mathrm{ex}}\right)$ denotes the interfacial exchange energy and the third and fourth terms are the energy of the uniaxial $\left(K_{U}\right)$ and the biaxial $\left(K_{C}\right)$ anisotropies. The last term describes the Zeeman energy in an external field $H$ with the $(\mathrm{Ga}, \mathrm{Mn})$ As saturation magnetization $M$. Due to the strong antiferromagnetic interface coupling between $\mathrm{Fe}$ and (Ga,Mn)As, the angle $\varphi_{1}=180^{\circ}$. According to the StonerWohlfarth coherent rotation model, ${ }^{30}$ one obtains, by energy minimization $\left(\frac{\partial^{2} E}{\partial \varphi_{2}^{2}}>0\right)$,

$$
H_{\mathrm{ex}}=-2 \sqrt{A K_{\mathrm{eff}}} / M t_{\mathrm{GMA}}
$$

as an expression for the exchange field. ${ }^{10}$

To compute the exchange field, we used literature data for $(\mathrm{Ga}, \mathrm{Mn}) \mathrm{As}$, in terms of the spin stiffness $A=4 \times$ $10^{-8} \mathrm{erg} / \mathrm{cm},{ }^{29}$ the uniaxial anisotropy constant $K_{U}=-1.1 \times$ $10^{3} \mathrm{erg} / \mathrm{cm}^{3}$, and the cubic anisotropy constant $K_{C}=2.2 \times$ $10^{3} \mathrm{erg} / \mathrm{cm}^{3}{ }^{31}$ Because of the strong cubic anisotropy we can consider $K_{\text {eff }} \approx K_{C} / 4 .^{29}$ The (Ga,Mn)As saturation magnetization was determined by SQUID to be $M=38 \mathrm{G}$ (at $T=12.5 \mathrm{~K}$ ) for $12 \% \mathrm{Mn}$ and $18 \mathrm{G}$ (at $T=10 \mathrm{~K}$ ) for $6 \%$ Mn (both $30 \mathrm{~nm}$ thickness). This yields for $t_{\mathrm{GMA}}=30 \mathrm{~nm}$ an exchange bias of $H_{\mathrm{ex}}=-82 \mathrm{Oe}$ at $12.5 \mathrm{~K}(12 \% \mathrm{Mn})$ and $H_{\mathrm{ex}}=-174 \mathrm{Oe}$ at $10 \mathrm{~K}(6 \%)$. Although this is only a very crude model, the calculated exchange bias shows reasonable agreement with our measurement on Fe/30 nm (Ga,Mn)As with $12 \%$, whereas it overestimates the exchange field (for the corresponding magnetization) of $\mathrm{Fe} / 30 \mathrm{~nm}(\mathrm{Ga}, \mathrm{Mn}) \mathrm{As}$ with $6 \% \mathrm{Mn}$ by a factor of 10 . In order to get a full understanding of whether this deviation is due to the limitations of the partial domain wall model, one would have to determine all material parameters like spin stiffness and (temperature-dependent) anisotropy constants for each individual sample, which was not in the focus of this work.

Further investigations were performed concerning the temperature dependence of the exchange bias field $H_{E}$. Figure 4(d) shows that the exchange field decreases nearly linearly with increasing temperature. At first glance, an increase of the exchange field is expected because of the reduced magnetization at higher temperatures. But, as already reported by Wilson et al. this discrepancy can be explained within the partial domain wall model, assuming the temperature dependence of the anisotropy, ${ }^{29,32,33}$ which may overcome that of the magnetization with increasing temperature. ${ }^{10}$

\section{Summary}

In summary, we present XMCD and SQUID data on a series of fully epitaxial $\mathrm{Fe} /(\mathrm{Ga}, \mathrm{Mn})$ As bilayers with different (Ga,Mn)As film thicknesses and Mn concentrations. We find an unusual transition from ferromagnetic to antiferromagntic coupling of the interfacial Mn moments to the Fe moments. The data can be corroborated with the help of $a b$ initio calculations where the influence of the increasing density of interstitial $\mathrm{Mn}$ at the interface is taken into account for thinner (Ga,Mn)As layers. In addition, we study exchange bias in these fully epitaxial bilayers and find rather strong ferromagnetic exchange bias. Using a simple partial domain wall model, the magnitude of the exchange bias can be estimated. Our findings may prove to be helpful when engineering the magnetization direction of thin (Ga,Mn)As layers, which can show induced ferromagnetism at room temperature due to the proximity polarization and which can be used for spin injection devices.

\section{ACKNOWLEDGMENTS}

This work has been supported by the Deutsche Forschungsgemeinschaft (DFG) via SFB689. We acknowledge ELETTRA for provision of synchrotron radiation facilities.
${ }^{1}$ T. Dietl, Nat. Mater. 9, 965 (2010).

${ }^{2}$ H. Macdonald, P. Schiffer, and N. Samarth, Nature (London) 4, 195 (2005).

${ }^{3}$ T. Dietl, H. Ohno, and F. Matsukura, Phys. Rev. B 63, 195205 (2001).

${ }^{4}$ A. Kaminski and S. Das Sarma, Phys. Rev. Lett. 88, 247202 (2002).

${ }^{5}$ K. W. Edmonds, P. Bogusławski, K. Y. Wang, R. P. Campion, S. N. Novikov, N. R. S. Farley, B. L. Gallagher, C. T. Foxon, M. Sawicki, T. Dietl, M. Buongiorno Nardelli, and J. Bernholc, Phys. Rev. Lett. 92, 037201 (2004).

${ }^{6}$ L. Chen, S. Yan, P. F. Xu, J. Lu, W. Z. Wang, J. J. Deng, X. Qian, Y. Ji, and J. H. Zhao, Appl. Phys. Lett. 95, 182505 (2009).
${ }^{7}$ T. Jungwirth, J. Sinova, J. Mašek, J. Kučera, and A. H. MacDonald, Rev. Mod. Phys. 78, 809 (2006).

${ }^{8}$ M. Zhu, M. J. Wilson, B. L. Sheu, P. Mitra, P. Schiffer, and N. Samarth, Appl. Phys. Lett. 91, 192503 (2007).

${ }^{9}$ M. Zhu, M. J. Wilson, P. Mitra, P. Schiffer, and N. Samarth, Phys. Rev. B 78, 195307 (2008).

${ }^{10}$ M. J. Wilson, M. Zhu, R. C. Myers, D. D. Awschalom, P. Schiffer, and N. Samarth, Phys. Rev. B 81, 045319 (2010).

${ }^{11}$ S. Mark, C. Gould, K. Pappert, J. Wenisch, K. Brunner, G. Schmidt, and L. W. Molenkamp, Phys. Rev. Lett. 103, 017204 (2009).

${ }^{12}$ F. Maccherozzi, M. Sperl, G. Panaccione, J. Minár, S. Polesya, H. Ebert, U. Wurstbauer, M. Hochstrasser, G. Rossi, G. Woltersdorf, 
W. Wegscheider, and C. H. Back, Phys. Rev. Lett. 101, 267201 (2008).

${ }^{13}$ M. Sperl, F. Maccherozzi, F. Borgatti, A. Verna, G. Rossi, M. Soda, D. Schuh, G. Bayreuther, W. Wegscheider, J. C. Cezar, F. Yakhou, N. B. Brookes, C. H. Back, and G. Panaccione, Phys. Rev. B 81, 035211 (2010).

${ }^{14}$ K. Olejnik, P. Wadley, J. A. Haigh, K. W. Edmonds, R. P. Campion, A. W. Rushforth, B. L. Gallagher, C. T. Foxon, T. Jungwirth, J. Wunderlich, S. S. Dhesi, S. A. Cavill, G. van der Laan, and E. Arenholz, Phys. Rev. B 81, 104402 (2010).

${ }^{15}$ C. Song, M. Sperl, M. Utz, M. Ciorga, G. Woltersdorf, D. Schuh, D. Bougeard, C. H. Back, and D. Weiss, Phys. Rev. Lett. 107, 056601 (2011).

${ }^{16}$ U. Wurstbauer, M. Sperl, D. Schuh, G. Bayreuther, J. Sadowski, and W. Wegscheider, J. Cryst. Growth 301, 260 (2007).

${ }^{17}$ U. Wurstbauer, M. Sperl, M. Soda, D. Neumaier, D. Schuh, G. Bayreuther, J. Zweck, and W. Wegscheider, Appl. Phys. Lett. 92, 102506 (2008).

${ }^{18}$ M. Reinwald, U. Wurstbauer, M. Döppe, W. Kipferl, K. Wagenhuber, H.-P. Tranitz, D. Weiss, and W. Wegscheider, J. Cryst. Growth 278, 690 (2005).

${ }^{19}$ H. Ebert and R. Zeller, SPR-TB-KKR package (2006), http://olymp. cup.uni-muenchen.de/ak/ebert/SPR-TB-KKR.

${ }^{20}$ S. H. Vosko, L. Wilk, and M. Nusair, Can. J. Phys. 58, 1200 (1980).

${ }^{21}$ M. Matsumoto, J. B. Staunton, and P. Strange, J. Phys.: Condens. Matter 2, 8365 (1990).

${ }^{22}$ W. H. Butler, Phys. Rev. B 31, 3260 (1985).
${ }^{23}$ T. J. Zega, Aubrey T. Hanbicki, S. C. Erwin, I. Zutić, G. Kioseoglou, C. H. Li, B. T. Jonker, and R. M. Stroud, Phys. Rev. Lett. 96, 196101 (2006).

${ }^{24}$ A. I. Liechtenstein, M. I. Katsnelson, V. P. Antropov, and V. A. Gubanov, J. Magn. Magn. Mater. 67, 65 (1987).

${ }^{25}$ A. Koeder, S. Frank, W. Schoch, V. Avrutin, W. Limmer, K. Thonke, R. Sauer, A. Waag, M. Krieger, K. Zuern, P. Ziemann, S. Brotzmann, and H. Bracht, Appl. Phys. Lett. 82, 3278 (2003).

${ }^{26}$ K. M. Yu, W. Walukiewicz, T. Wojtowicz, J. Denlinger, M. A. Scarpulla, X. Liu, and J. K. Furdyna, Appl. Phys. Lett. 86, 042102 (2005).

${ }^{27}$ D. Mauri, H. C. Siegmann, P. S. Bagus, and E. Kay, J. Appl. Phys. 62, 3047 (1987).

${ }^{28}$ Z. J. Guo, J. S. Jiang, J. E. Pearson, S. D. Bader, and J. P. Liu, Appl. Phys. Lett. 81, 2029 (2002).

${ }^{29}$ A. Sugawara, H. Kasai, A. Tonomura, P. D. Brown, R. P. Campion, K. W. Edmonds, B. L. Gallagher, J. Zemen, and T. Jungwirth, Phys. Rev. Lett. 100, 047202 (2008).

${ }^{30}$ E. C. Stoner and E. P. Wohlfarth, Philos. Trans. R. Soc. London A 240, 599 (1948).

${ }^{31}$ F. Hoffmann, G. Woltersdorf, W. Wegscheider, A. Einwanger, D. Weiss, and C. H. Back, Phys. Rev. B 80, 054417 (2009).

${ }^{32}$ M. Cubukcu, H. J. von Bardeleben, K. Khazen, J. L. Cantin, M. Zhu, M. J. Wilson, P. Schiffer, and N. Samarth, J. Appl. Phys. 105, 07C506 (2009).

${ }^{33}$ M. Sawicki, F. Matsukura, A. Idziaszek, T. Dietl, G. M. Schott, C. Ruester, C. Gould, G. Karczewski, G. Schmidt, and L. W. Molenkamp, Phys. Rev. B 70, 245325 (2004). 